\title{
Activation of Ras/Raf protects cells from melanoma differentiation-associated gene-5-induced apoptosis
}

\author{
L Lin $^{1,6}$, Z Su $^{1,6}$, IV Lebedeva ${ }^{1,6}$, P Gupta ${ }^{1}$, H Boukerche ${ }^{1}$, T Rai $^{1}$, \\ GN Barber ${ }^{2}, P$ Dent ${ }^{3}$, D Sarkar ${ }^{1}$ and PB Fisher ${ }^{*, 1,4,5}$ \\ 1 Department of Pathology, Herbert Irving Comprehensive Cancer Center, \\ Columbia University Medical Center, College of Physicians and Surgeons, \\ New York, NY 10032, USA \\ 2 Department of Microbiology and Immunology, Sylvester Comprehensive \\ Cancer Center, University of Miami School of Medicine, Miami, FL 33136, \\ USA \\ 3 Department of Radiation Oncology, Virginia Commonwealth University, \\ Richmond, VA 23298, USA \\ ${ }^{4}$ Department of Neurosurgery, Herbert Irving Comprehensive Cancer Center, \\ Columbia University Medical Center, College of Physicians and Surgeons, \\ New York, NY 10032, USA \\ ${ }^{5}$ Department of Urology, Herbert Irving Comprehensive Cancer Center, \\ Columbia University Medical Center, College of Physicians and Surgeons, \\ New York, NY 10032, USA \\ 6 These authors contributed equally to this work \\ * Corresponding author: PB Fisher, Department of Pathology, Columbia \\ University Medical Center, College of Physicians and Surgeons, BB-1501, 630 \\ West 168th Street, New York, NY 10032, USA. Tel: +1 212305 3966; \\ Fax: + 1212305 8177; E-mail: pbf1@ columbia.edu
}

Received 24.8.05; revised 14.2.06; accepted 15.2.06; published online 31.3.06 Edited by M Blagosklonny

\begin{abstract}
Melanoma differentiation-associated gene-5 (mda-5) was the first molecule identified in nature whose encoded protein embodied the unique structural combination of an $\mathrm{N}$-terminal caspase recruitment domain and a C-terminal DEXD/H RNA helicase domain. As suggested by its structure, cumulative evidences documented that ectopic expression of mda-5 leads to growth inhibition and/or apoptosis in various cell lines. However, the signaling pathways involved in mda-5-mediated killing have not been elucidated. In this study, we utilized either genetically modified cloned rat embryo fibroblast cells overexpressing different functionally and structurally distinct oncogenes or human pancreatic and colorectal carcinoma cells containing mutant active ras to resolve the role of the Ras/Raf signaling pathway in mda-5-mediated growth inhibition/apoptosis induction. Rodent and human tumor cells containing constitutively activated Raf/Raf/MEK/ERK pathways were resistant to mda5 -induced killing and this protection was antagonized by intervening in this signal transduction cascade either by directly inhibiting ras activity using an antisense strategy or by targeting ras-downstream factors, such as MEK1/2, with the pharmacological inhibitor PD98059. The present findings provide a further example of potential cross-talk between growth-inhibitory and growth-promoting pathways in which the ultimate balance of these factors defines cellular homeostasis, leading to survival or induction of programmed cell death.
\end{abstract}

\author{
Cell Death and Differentiation (2006) 13, 1982-1993. \\ doi:10.1038/sj.cdd.4401899; published online 31 March 2006
}

Keywords: oncogenic transformation; ras/raf signaling; apoptosis; $m d a-5$

\begin{abstract}
Abbreviations: Ad, adenovirus; APC, allophycocyanine; AS, antisense; CARD, caspase recruitment domain; Cdkls, cyclindependent kinase inhibitors; CREF, cloned rat embryo fibroblasts; ERK, extracellular signal-regulated kinase; IFN- $\beta$, fibroblast (beta) interferon; MAPK, mitogen-activated protein kinase; mda-5, melanoma differentiation-associated gene-5; MEK, MAPK/ERK kinase; MEZ, mezerein; m.o.i., multiplicity of infection; MTT, 3-(4,5dimethylthiazol-2-yl)-2,5-diphenyltetrazolium bromide; pfu, plaqueforming units; PI3K, phosphatidylinositol-3-kinase; PKC, protein kinase C; SAPK/JNK, stress-activated protein kinase/c-Jun $\mathrm{N}$ terminal kinase.
\end{abstract}

\section{Introduction}

In human melanoma cells, combinatorial treatment with interferon- $\beta$ and mezerein, a protein kinase $C$ (PKC)-activating compound, leads to terminal differentiation characterized by a rapid and irreversible loss in proliferation, changes in antigenic phenotypes and gene expression, and a decrease in tumorigenic potential in nude mice. ${ }^{1-3}$ Melanoma differentiation-associated gene-5 (mda-5) was initially identified by subtraction hybridization as an upregulated transcript in terminally differentiated $\mathrm{HO}-1$ human melanoma cells. ${ }^{3,4}$ The encoded MDA-5 protein was recognized as the first molecule with a unique structural combination: an $\mathrm{N}$-terminal caspase recruitment domain (CARD) and a C-terminal putative RNA helicase domain. ${ }^{3,4}$ Our previous studies demonstrate that $m d a-5$ is an early interferon inducible gene, and its expression requires an intact interferon signaling pathway, as there is no detectable expression of mda-5 in cells containing defects in the Jak/Stat signaling pathway upon interferon treatment. ${ }^{4}$

Based on the presence in the MDA-5 protein of a CARD motif, a proapoptotic element present in many caspases that facilitates cell death through homophilic protein-protein interactions, we proposed that mda-5 might play a role in mediating programmed cell death. ${ }^{3}$ This hypothesis was later proven correct. ${ }^{4}$ When ectopically expressed, mda-5 promotes growth inhibition in many tumor cells, as demonstrated by both cell viability and colony formation assays. Induction of membrane blebbing, formation of an internucleosomal DNA ladder on agarose gels and Annexin $\mathrm{V}$ binding on the exposed phosphatidylserine of dying cells, all characteristics of apoptosis, provide direct confirmation that mda-5-induced cell death occurs by apoptosis. ${ }^{4}$ The ability of mda-5 to mediate apoptosis in a variety of tumor cells embodying various genetic mutations, including $\mathrm{Rb}, \mathrm{p} 53$ and bax, indicates that mda-5-induced killing is independent of many 
key cell death-regulating factors (unpublished data). The mouse homolog of mda-5 (Helicard) has also been shown to cause apoptosis by facilitating DNA fragmentation in in vitro experiments. ${ }^{5}$ However, the underlying mechanism of $m d a-5-$ induced and Helicard-induced apoptosis is presently unknown.

The Ras/Raf/MEK/ERK pathway plays a pivotal role in cell proliferation and prevention of apoptosis. ${ }^{6}$ Ras, a GTPbinding protein, is a common upstream activator of several signaling pathways, including Raf/MEK/ERK. ${ }^{6-8}$ Raf is a serine/threonine kinase and can be activated through recruitment to the plasma membrane by interacting with Ras, followed by dimerization between Raf proteins, and phosphorylation of different Raf domains. Additionally, impairment of ras activity hinders raf activation. ${ }^{6,9-11}$ Aberrant activation of this pathway is frequently linked with tumor progression and malignancy, which can contribute to the low efficacy of chemotherapy in patients. ${ }^{6}$ Mutations leading to constitutive activation of ras have been found in approximately $30 \%$ of all human cancers. ${ }^{12}$ For example, ras mutations contribute as a molecular defect to $85-90 \%$ of pancreatic cancers and about $50 \%$ of colorectal cancers. ${ }^{13-16}$ Although extensive evidence has confirmed an effect of ras on tumorigenesis in immortal cell lines, ${ }^{17}$ other studies have also demonstrated antiproliferative influences of oncogenic ras in nontransformed cells via induction of premature $\mathrm{G}_{1}$ arrest and senescence by $\mathrm{p} 15^{\text {Ink4b }}$ and $\mathrm{p} 16^{\text {Ink4a }}$ through the Raf/MEK/ERK pathway. ${ }^{18}$

Our current study expands on our previous findings $s^{3,4}$ corroborating the ability of $m d a-5$ to induce apoptosis when overexpressed using a recombinant replication-incompetent adenovirus, Ad.mda-5, in a spectrum of tumor cell lines. Additionally, we have examined the effects of specific oncogenic mutations, including $H P V-18$, a specific temperature-sensitive mutant of type 5 adenovirus (H5hr1), src, ras and raf, on mda-5-mediated killing in an immortal normal rat embryo fibroblast cell line (CREF), which was developed in our laboratory to analyze the role of discrete genetic changes in the process of cancer progression. ${ }^{19,20}$ CREF cells exhibit properties of untransformed rat embryo cells, including contact inhibition, failure to grow in an anchorage-independent manner in agar and lack of tumorigenic potential when injected into athymic nude mice or syngeneic Fischer rats. ${ }^{19-21}$ CREF cells are susceptible to oncogenic transformation by different cellular and viral oncogenes, including Ha-ras, v-src, v-raf, HPV-18 and H5hr1. ${ }^{15,20-23}$ Unlike human tumor cells, which frequently harbor numerous mutations in several genes, CREF cells and their single oncogene-transformed counterparts reflect a homogeneous genetic background, thereby facilitating a precise determination of the role of specific oncogenic changes in susceptibility to $m d a-5$-induced apoptosis. Based on these considerations, we used this welldefined system to examine the effects of ras and raf as well as other oncogenes, including H5hrl, HPV and src, on mda-5induced killing. Among the different transformed variants of CREF cells, only ras-and raf-transformed CREF cells (CREFras and CREF-raf) were protected from cell death induced by mda-5. Based on these observations, studies were extended to human tumor cells, including colorectal and pancreatic carcinoma cells with a well-defined ras status, again highlighting a role for this genetic change in mediating resistance to mda-5. A direct functional relationship between ras activation and protection of tumor cells from mda-5-induced death was confirmed by inhibiting ras function using antisense or genetic deletion approaches, thereby eliciting sensitivity to mda-5-induced apoptosis. Furthermore, PD98059, a specific inhibitor of MEK1/2 that has been shown to inhibit MEK/ERK pathway activation, also diminished the protection of mutated active ras in preventing mda-5-induced cell death. These studies support an antiapoptotic role of the Ras/Raf/MEK/ ERK signaling pathway in regulating mda-5-mediated death of tumor cells.

\section{Results}

\section{Ad.mda-5 induces growth suppression and apoptosis in parental CREF cells, but not in mutant ras- or raf-transformed CREF cells}

Parental nontransformed and different oncogene-transformed CREF cells were infected with either Ad.null (a recombinant adenovirus lacking the mda-5 gene) or Ad.mda-5 at a multiplicity of infection (m.o.i.) of 50 plaque-forming units (pfu) per cell, as described in Materials and Methods. After $48 \mathrm{~h}$, adenovirus-mediated expression of $m d a-5$ was detected at the mRNA and protein levels by Northern and Western blot analyses, respectively (Figure 1a). Parental and transformed CREF cells produced both mda- 5 mRNA and protein after Ad.mda-5 infection, but not following Ad.null infection (Figure 1a). A quantitative analysis of the level of mda-5 expression using densitometer scanning confirmed comparable levels of $m d a-5$ in the different CREF cells (data not shown).

MTT (3-(4,5-dimethylthiazol-2-yl)-2,5-diphenyltetrazolium bromide) assays were used to determine cell proliferation and viability. At 5 days after Ad. mda- 5 infection (100 pfu/cell), significant growth inhibition and/or viability decreases were apparent in CREF, CREF-H5hrl, CREF-HPV and CREF-src cells, but not in CREF-raf and CREF-ras cells (Figure 1b). Ad.mda-5-infected CREF, CREF-H5hrl, CREF-HPV and CREF-src cells grew at a rate of $49,46,66$ and $58 \%$ of uninfected cells, respectively (Figure 1b). Ad.null infection did not affect cell growth in any of the cell lines in comparison with their uninfected counterparts, ruling out the possibility that adenoviral infection at $100 \mathrm{pfu} / \mathrm{cell}$ might have contributed to this growth inhibition and/or viability decrease. Colony formation assays further demonstrated that Ad.mda-5 infection at $100 \mathrm{pfu} / \mathrm{cell}$ led to significant decreases in colony numbers in CREF, CREF-H5hrl, CREF-HPV and CREF-src cells, but not in CREF-raf or CREF-ras cells (Figure 1c). The colonies formed by CREF, CREF-H5hrl, CREF-HPV and CREF-src after Ad.mda-5 infection accounted for 60, 46, 66 and $58 \%$ of colonies formed by uninfected cells, respectively. Ad.null infection alone did not change the number of colonies formed in any cell type as compared to uninfected control cells (Figure 1c). Growth inhibition shown by MTT or colony formation assays may result from cell growth arrest or cell death. As mda-5 is known to induce apoptosis, the mechanism of growth inhibition in CREF, CREF-H5hrl, CREF-HPV and CREF-src cells after Ad.mda-5 infection was examined using apoptosis assays. At 2 days after infection with either 


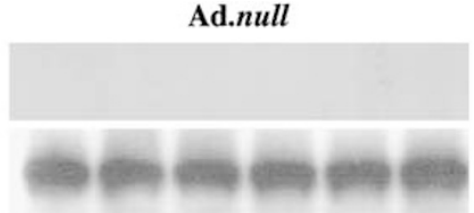

CREF HPV Raf Ras H5hrl Src
Ad. $m$ da-5

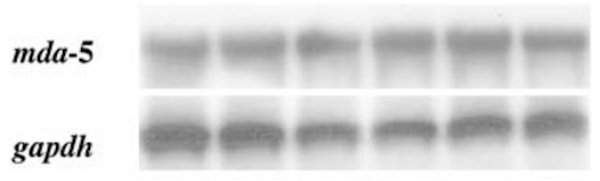

CREF HPV Raf Ras H5hrl Src

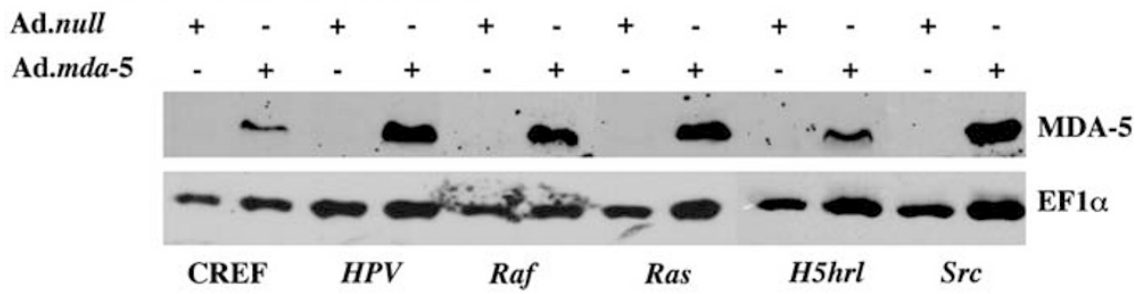

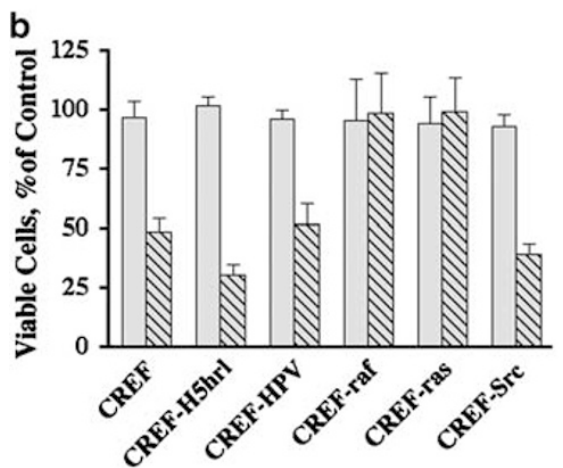

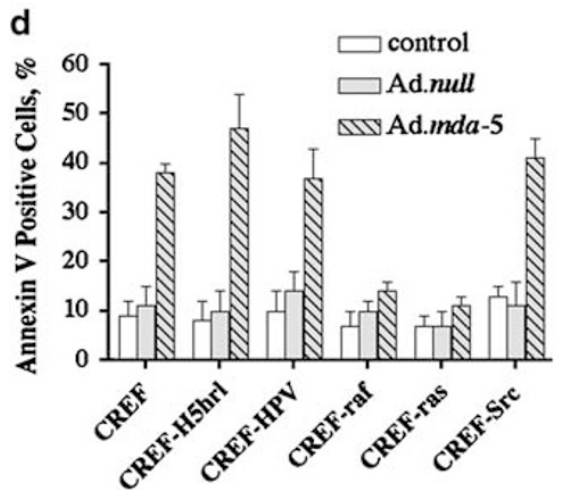

C

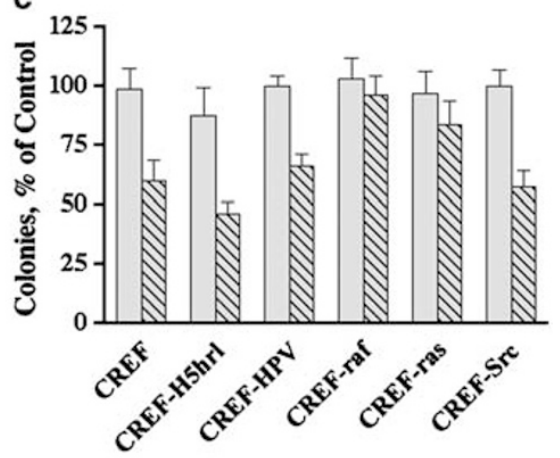

e

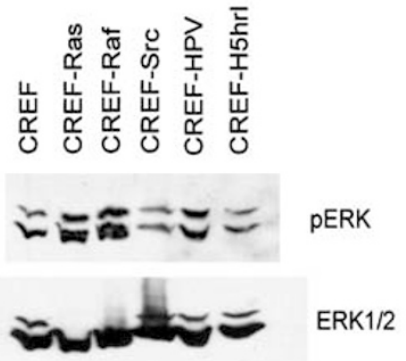

Figure 1 Ras- and raf-transformed CREF cells are resistant to mda-5-induced cell death. Cells were infected with Ad.null or Ad.mda-5 at an $\mathrm{m} .0 . \mathrm{i}$. of 100 pfu/cell. (a) (Top) mRNA levels of mda-5 determined by Northern blot analysis. (Bottom) Protein levels of MDA-5 determined by Western blotting analysis. (b) Cells plated at 1000 cells per well in 96-well plates on the previous day were infected with either Ad.null or Ad. mda-5, and cell viability was measured by MTT assay on the fifth day after infection. (c) Effect of Ad.mda-5 infection on the cloning (colony-forming) ability of CREF clones. Cells infected on the previous day were replated at 300 cells/60-mm plate. (d) CREF clones plated on six-well plates (20000 cells/well) on the previous day were infected with Ad.null or Ad.mda-5 and Annexin V binding was determined $48 \mathrm{~h}$ later. (e) The level of phospho- and total ERK in the indicated cell lines was determined by Western blot analysis

Ad.null or Ad.mda-5 at 100 pfu/cell, parental and transformed CREF cells were incubated with Annexin V-allophycocyanine (APC) and positively stained cells, indicative of apoptotic cells, were measured by flow cytometry. Although there was some level of spontaneous apoptosis (up to $13 \%$ in CREF-src cells) in uninfected control cells, Ad.mda-5 infection resulted in 38, 47, 37 and $41 \%$ Annexin V-positive cells in CREF, CREF-H5hrl, CREF-HPV and CREF-src cells, respectively (Figure 1d). Ad.null infection did not cause any statistically relevant level of apoptosis above the basal level in any of the
CREF cells. In contrast, CREF-raf and CREF-ras cells displayed no apoptosis induction following Ad.mda-5 infection (100 pfu/cell) when compared with uninfected or Ad.nullinfected cells (Figure 1d). The level of activation of pathways downstream of ras or raf was analyzed by Western blot analysis for phosphorylated ERK in different oncogenetransformed CREF cell lines. Higher phospho-ERK level was observed in CREF-ras and CREF-raf cells than the other cell lines (Figure 1e). The level of total ERK was similar in all the cell lines. These results argue that activation of ras or raf 
protects rodent cells from apoptosis induction resulting from overexpression of $m d a-5$.

To further confirm an antiapoptotic role of ras/raf in mda-5induced cell death in CREF cells, we used a replicationincompetent adenovirus expressing antisense (AS) K-ras (Ad.Kras(AS)) to block ras function. As shown by MTT assay in Figure 2a, none of the three Ads, Ad.null, Ad.mda-5 or Ad.Kras(AS), when used alone at 100 pfu/cell, caused growth suppression or viability loss in CREF-raf or CREF-ras cells. However, the combination of Ad.mda-5 plus Ad.Kras(AS) applied at $50 \mathrm{pfu} / \mathrm{cell}$ each led to induction of growth inhibition in CREF-ras cells to a comparable level ( $56 \%$ of untreated) as that found in CREF cells infected with Ad. mda- 5 alone (59\% of untreated) or combined with Ad.Kras(AS) (52\% of untreated control). In contrast, the combinatorial treatment of CREF-raf with Ad.mda-5 and Ad.Kras(AS) failed to suppress cell proliferation, further confirming that raf activation is downstream of ras activation (Figure 2a). Annexin V staining demonstrated that the induction of growth inhibition in CREF cells by Ad.mda-5 and in CREF-ras cells by Ad.mda- 5 plus Ad.Kras(AS) resulted from apoptosis, and neither Ad.mda-5 alone nor in combination with Ad.Kras(AS) promoted apoptosis in CREF-raf cells (Figure $2 b$ ).

In agreement with the findings that $v$-ras- or $v$-raftransformed cells display elevated activation of the mitogenactivated protein kinase (MAPK) pathway, ${ }^{24}$ the expression of oncogenic forms of ras and raf in CREF cells also activated this pathway. ${ }^{15}$ To resolve whether ras/raf activation exerts its antiapoptotic effect through the MEK/ERK pathway, we used PD98059, a pharmacological inhibitor of MEK1/2 capable of blocking the ras/rafMEK/ERK signaling pathway. The mda-5induced killing effect in the presence or absence of PD98059 was determined by colony formation assay. As expected, when PD98059 was applied along with Ad.mda-5, colony formation was reduced significantly, not only in CREF-ras ( $49 \%$ of uninfected or Ad.null-infected cells) but also in CREFraf cells (52\% of uninfected or Ad.null-infected cells), whereas Ad.mda-5 alone did not cause any significant decrease in colony formation in either cell type (Figure 2c). In CREF parental cells, Ad.mda-5 resulted in a similar decrease in colony numbers regardless of whether PD98059 was present or absent (Figure $2 \mathrm{c}$ ). The combinatorial treatment with Ad. $m d a-5$ and Ad.Kras(AS) promoted colony reduction in CREF-ras, but not in CREF-raf (Figure 2c), which was consistent with MTT and Annexin V data (Figure $2 a$ and b).

Experiments were performed to determine whether the antagonistic effects of ras and raf were mediated by their ability to modulate the level of overexpressed MDA-5 protein. Western blot analysis of CREF, CREF-raf and CREF-ras cells infected with Ad.mda-5 alone or in combination with Ad.Kras(AS), or in the presence of PD98059, displayed similar levels of MDA-5 protein (Figure 3), indicating that inhibition of Raf/Raf/MEK/ERK by either AS K-ras or PD98059 did not modulate MDA-5 protein levels. Employment of specific K-Ras and c-Raf antibodies revealed that CREF-ras contains the highest level of K-ras protein, and CREF-raf has the most abundant expression of c-Raf protein among the three cell lines examined, thus confirming the genetic authenticity of these cell lines (Figure 3). Ad.Kras(AS) infection reduced ras protein levels in CREF-ras cells
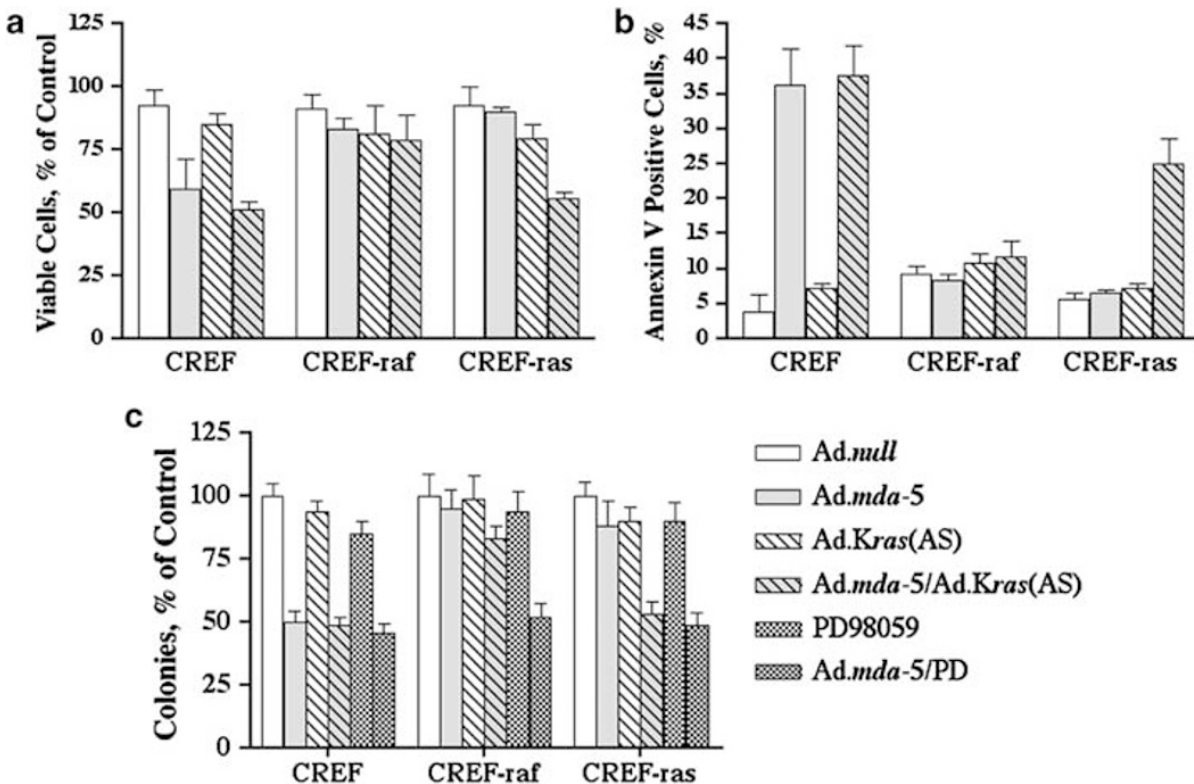

Figure 2 Inhibition of Ras/Raf/MEK/ERK activation by AS K-ras sensitizes CREF-ras, but not CREF-raf, to mda-5-induced growth inhibition and apoptosis. (a) The indicated cells plated at 1000 cells per well in 96-well plates on the previous day were infected with Ad.null, Ad.mda-5 or Ad.Kras(AS) alone at 100 pfu/cell or infected with Ad. $m d a-5$ plus Ad.Kras(AS) at 50 pfu/cell each. Cell viabilities were measured by MTT assays on the fifth day after infection. (b) The indicated cells were plated in six-well plates (20000 cells/well) on the previous day and infected with Ad.null, Ad.mda-5 or Ad.Kras(AS) alone at 100 pfu/cell, or infected with Ad.mda-5 plus Ad.Kras(AS) at $50 \mathrm{pfu} / \mathrm{cell}$ of each virus. After 2 days, cells were stained with Annexin V-APC and analyzed by flow cytometry. (c) Colony (clone)-forming ability was determined after infection with Ad.mda-5 in the presence or absence of Ad.Kras(AS) or PD98059. CREF, CREF-raf and CREF-ras cells were infected with Ad.null, Ad.mda-5, or Ad.Kras(AS) alone at $100 \mathrm{pfu} / \mathrm{cell}$, or with Ad.mda-5 plus Ad.Kras(AS) at 50 pfu/cell of each virus, or with Ad. $m d a-5$ (100 pfu/cell) in the presence of PD98059 (10 $\mu \mathrm{M})$ for $24 \mathrm{~h}$ and replated at $300 \mathrm{cells} / 60-\mathrm{mm}$ plate for colony formation assay 


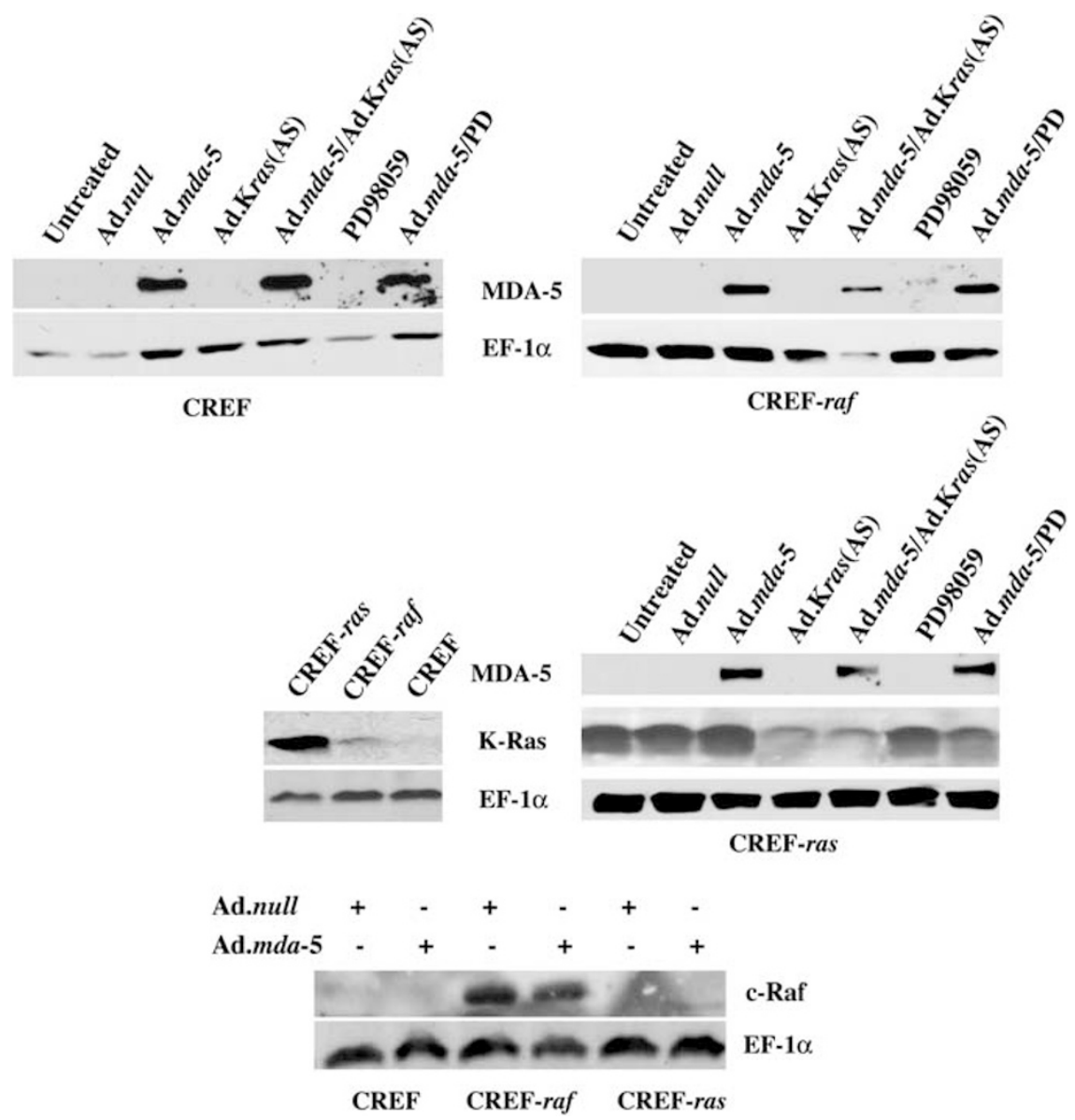

Figure 3 Expression of MDA-5, K-Ras and c-Raf proteins in CREF, CREF-raf and CREF-ras cells following Ad.mda-5 infection in the presence or absence of Ad.Kras(AS) or PD98059. Cells were infected with Ad.null, Ad.mda-5 or Ad.Kras(AS) alone at 100 pfu/cell, or with Ad.mda-5 plus Ad.Kras(AS) at 50 pfu/cell of each virus, or with Ad.mda-5 (100 pfu/cell) in the presence of PD98059 $(10 \mu \mathrm{M})$ for $48 \mathrm{~h}$. Proteins were extracted and resolved on SDS-PAGE, followed by immunoblotting with MDA-5, K-Ras or Raf-1 antibodies. Expression of EF-1 $\alpha$ was used to normalize sample loading

(Figure 3), which correlated with the reversal of mda-5-induced killing in these cells when infected with both Ad.mda-5 and Ad.Kras(AS) (Figure 2). It should be noted that Ad.null and Ad.mda-5 infection did not alter the level of Raf protein in CREF-raf, CREF or CREF-ras cells (Figure 3). In total, these findings suggest that the ras/raf/MEK/ERK signaling pathway is pivotal in protecting rodent cells from $m d a-5-$ mediated lethality.

\section{Mutant ras activation protects human pancreatic carcinoma cells from Ad.mda-5-induced growth inhibition and apoptosis}

Studies were performed to determine if ras protection against mda-5-induced killing in rodent CREF cells represents a general phenomenon that also extends to human cancer cells. To initially test this possibility, we used four pancreatic tumor cell lines, BxPC-3 cells which contain wild-type K-ras and PANC-1, MIA PaCa-2 and AsPC-1 cells that contain mutant active $\mathrm{K}$-ras. These cell lines have been intensively studied and well-characterized in our laboratory and by others. ${ }^{16,25}$

We initially examined the effect of Ad.mda-5 (100 pfu/cell) infection on cell growth by MTT assays. The three pancreatic tumor cells, PANC-1, MIA PaCa-2 and AsPC-1, expressing mutant active K-ras did not display significant levels of growth inhibition and/or cell viability loss following a single infection with either Ad.mda-5 or Ad.Kras(AS) in comparison with Ad.null-infected cells or following treatment with PD98059 (Figure 4a). However, a combination treatment of these cells with both Ad.mda-5 and Ad.Kras(AS) or Ad.mda-5 and PD98059 resulted in significant decreases in cell viability. Among these cells, PANC-1 were most susceptible to Ad.mda-5 plus Ad.Kras(AS) infection or Ad.mda-5 and PD98059 treatment, demonstrating an 90\% decrease in cell viability in comparison with Ad.null-infected cells (Figure 4a). Infection of MIA Paca-2 and AsPC-1 cells with Ad. mda-5 and Ad.Kras(AS) resulted in an $\sim 60$ and $\sim 30 \%$ decrease in cell viability, respectively, when compared with their Ad.null-infected counterparts, whereas Ad.mda-5 and PD98059 treatment resulted in an $\sim 70$ decrease in cell viability in both these cells (Figure 4a). In contrast, in BxPC-3 cells manifesting wild-type K-ras, neither Ad.mda-5 alone nor Ad.mda-5 plus Ad.Kras(AS) or Ad.mda-5 and PD98059 treatment affected growth, indicating that the transformation of this cancer cell line may be ras-independent and that 

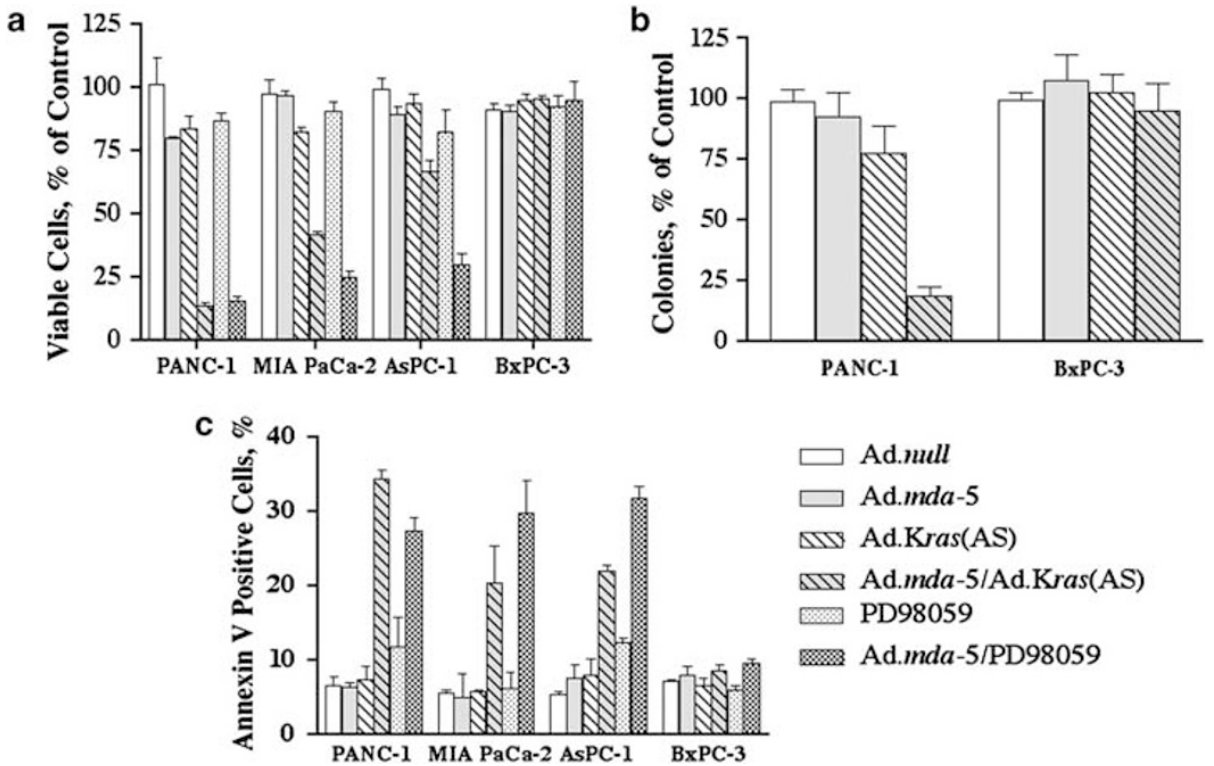

Figure 4 Inhibition of K-ras activity by adenovirus expressing AS K-ras or by treatment with PD98059 renders mutated K-ras human pancreatic carcinoma cells susceptible to mda-5-induced growth suppression and apoptosis. BxPC-3, PANC-1, MIA PaCa-2 and AsPC-1 were infected with Ad.null, Ad.mda-5 or Ad.Kras(AS) alone at 100 pfu/cell, or with Ad.mda-5 plus Ad.Kras(AS) at 50 pfu/cell of each virus or infected with Ad.mda-5 plus treated with PD98059 (10 $\mu \mathrm{M})$. (a) Cells plated at 1000 cells per well in 96-well plate on the previous day were infected with the indicated virus or treated with PD98059, and cell viabilities were measured on the fifth day after infection. (b) BxPC-3 and PANC-1 were infected as described for panel a for $24 \mathrm{~h}$ and replated at $500 \mathrm{cells} / 60$-mm plate. (c) Cells were plated in six-well plate (20000 cells/well) on the previous day, infected with the indicated viruses or treated with PD98059, and analyzed after 2 days by FACS for Annexin V staining

this pathway also protects from mda-5-induced lethality (Figure 4a).

Clonal assays further documented that combined infection with Ad.mda-5 and Ad.Kras(AS) at 50 pfu/cell promoted a dramatic decrease in colony numbers in PANC-1 cells expressing mutant $\mathrm{K}$-ras, but not in BxPC-3 cells containing wt K-ras (Figure 4b). Although Ad.mda-5 infection alone at $100 \mathrm{pfu} / \mathrm{cell}$ did not change the number of colonies formed in PANC-1 cells, the combinatorial infection with Ad.mda-5 and Ad.Kras(AS) decreased the colony-forming ability by $83 \%$ in comparison with uninfected control cells (Figure $4 \mathrm{~b}$ ). Neither Ad.mda-5 alone nor Ad.mda-5 plus Ad.Kras(AS) elicited a significant effect on clonigenicity in BxPC-3 cells, which corroborates the data obtained using MTT assays (Figure 4a).

Annexin $\mathrm{V}$ staining followed by fluorescence-activated cell sorting (FACS) analysis revealed that the growth inhibition induced by combined infection with Ad. mda-5 and Ad.Kras(AS) was due to apoptosis (Figure $4 \mathrm{c}$ ). In parallel with the viability assays, PANC-1 cells displayed more apoptotic cells (36\%) than MIA PaCa-2 (16\%) and AsPC-1 (21\%) cells following Ad.mda-5 plus Ad.Kras(AS) infection (Figure 4c). Ad.mda-5 and PD98059 treatment resulted in $\sim 25-30 \%$ apoptosis in these three cell lines. These findings were recapitulated by using siRNA specific for MEK1 and MEK2 (data not shown). There was no evidence of apoptosis in wt K-ras expressing BxPC-3 cells, even after combined treatment with Ad.mda-5 and Ad.Kras(AS) (Figure 4b).

Experiments were performed to explore the mechanism underlying the resistance of pancreatic carcinoma cells to mda-5. Infection of all four pancreatic carcinoma cells with Ad.mda-5 (100 pfu/cell), alone or in combination with
Ad.Kras(AS), resulted in mda-5 mRNA (Figure 5a). The relative levels of mda-5 mRNA were higher in PANC- 1 and MIA PaCa-2 cells with lower levels in BxPC-3 cells and lowest levels in AsPC-1 cells. In contrast, infection with only Ad.mda-5 did not result in detectable MDA-5 protein in any of these pancreatic carcinoma cells (Figure $5 b$ ). However, when the three mutant $\mathrm{K}$-ras pancreatic cancer cell lines (PANC-1, MIA PaCa-2 and AsPC-1), but not wild-type K-ras BxPC-3 cells, were infected with a combination of Ad.mda-5 and Ad.Kras(AS) MDA-5 protein was detected. This effect was associated with a marginal increase in mda-5 mRNA in PANC-1, MIA PaCa-2 and AsPC-1 cells, most evident in AsPC-1 cells. In contrast, no change in mda-5 mRNA was apparent in BxPC-3 following co-infection with Ad. mda-5 and Ad.Kras(AS). The levels of ras proteins in the pancreatic tumor cells were measured by Western blotting using anti-ras antibody, confirming that the Ad.Kras(AS) virus was able to decrease levels of K-ras in all four cell types (Figure $5 \mathrm{~b}$ ). The ability of this virus to decrease K-ras protein levels in mutant and wild-type K-ras cells was predicted and relates to the fact that the antisense construct was designed to target the initiation codon of this gene, thereby displaying no preferential activity toward mutant versus wild-type K-ras. This interesting finding suggests that a 'translational block' for mda- 5 exists in mutant K-ras pancreatic cancer cells, which can be ablated by blocking K-ras expression in a manner similar to that observed with the novel apoptosis-inducing cytokine gene mda-7/IL-24 in these cells. ${ }^{16,25}$ An additional mechanism such as increased degradation of protein might also be involved in regulating MDA-5 protein level in pancreatic cancer cells. Initial studies using proteasome inhibitors show an increase in the level of MDA-5 protein (data not shown), indicating that 
a

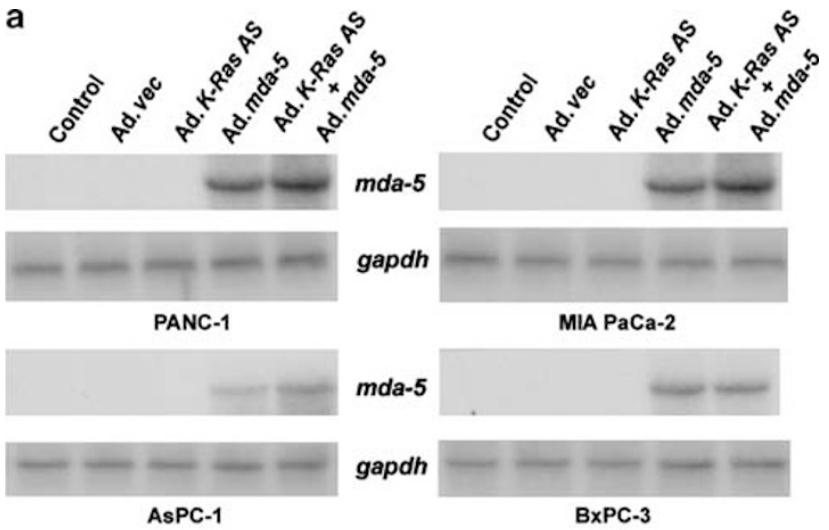

b
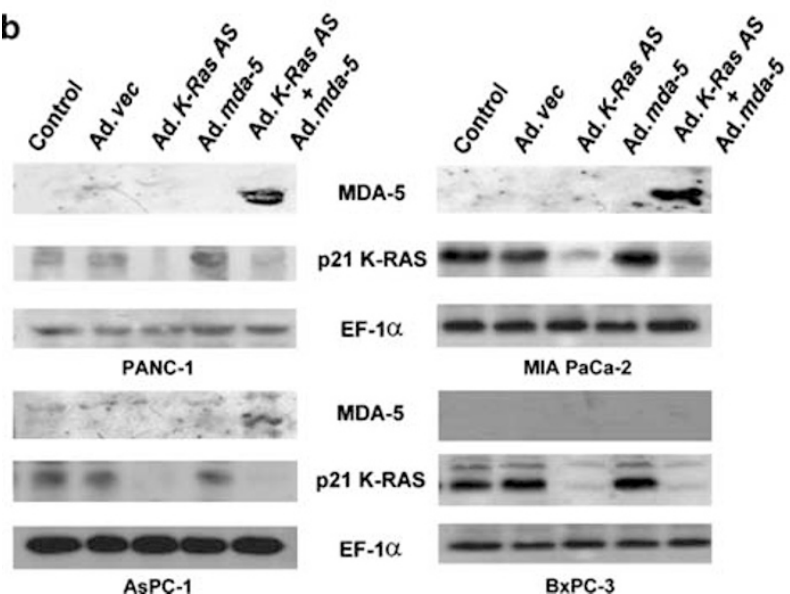

Figure 5 Expression of $m d a-5$ mRNA and MDA-5 and K-Ras proteins in pancreatic carcinoma cells following Ad.mda- 5 infection in the presence or absence of Ad.Kras(AS). (a) The indicated cell type was uninfected or infected with $100 \mathrm{pfu} / \mathrm{cell}$ with the indicated virus and $m d a-5$ and gapdh mRNA levels were determined $48 \mathrm{~h}$ later by Northern blotting. (b) Cells were infected with the indicated virus (total m.o.i. was $100 \mathrm{pfu} / \mathrm{cell}$ ) for $48 \mathrm{~h}$ and protein levels of MDA-5, p21 K-Ras and EF-1 $\alpha$ were determined by Western blotting using appropriate antibodies

multiple mechanisms control MDA-5 protein expression level in these cells.

\section{Mutant ras activation protects human colorectal carcinoma cells from Ad.mda-5-induced growth inhibition and apoptosis}

After confirming a protective effect of $\mathrm{K}$-ras from mda-5induced killing in human pancreatic tumor cells, we further tested this protection in human colorectal cancer cells, the second leading human cancer containing ras mutations. ${ }^{14,26}$ Two sets of human colorectal tumor cell lines were utilized, HCT 116 and Hkh2, and DLD-1 and DKO-4. ${ }^{27}$ Among these cell types, HCT 116 and DLD-1 contain mutated active $\mathrm{K}$-ras, whereas Hkh2 and DKO-4 are respective derivatives with their K-ras alleles knocked out by homologous recombination. ${ }^{27}$

As many colorectal carcinoma cells are sensitive to adenoviral infection (unpublished data), a total viral input of $25 \mathrm{pfu} / \mathrm{cell}$ was used. We first examined cell viability by
MTT assays. Compared with uninfected cells, Ad.null at $25 \mathrm{pfu} / \mathrm{cell}$ did not significantly affect cell growth in HCT 116, Hkh2, DLD-1 or DKO-4 cells (Figure 6a). Although Ad. $m d a-5$ (25 pfu/cell) resulted in significant growth inhibition in both K-ras-deleted cells, Hkh2 and DKO-4, demonstrating the cell viability of only 57 and $58 \%$ of their uninfected (data not shown) or Ad.null-infected counterparts, respectively, it did not lead to any significant decrease in cell viability in either of the K-ras constitutively active cells, HCT 116 or DLD-1 cells (Figure 6a). In HCT 116 and Hkh2, Ad.Kras(AS) infection at $25 \mathrm{pfu} / \mathrm{cell}$ alone caused a significant decrease in cell growth that was 70 and $42 \%$ of the uninfected cells, respectively (Figure 6a). Ad.Kras(AS) only marginally inhibited DLD-1 cell growth, whereas it produced an $\sim 41 \%$ decrease in cell viability in DKO-4 cells (Figure 6a). The inhibitory effect elicited by Ad.Kras(AS) may result from the inhibition of K-ras in HCT 116, and inhibition of $\mathrm{H}$-ras by AS K-ras owing to the homology between different ras isoforms in Hkh2 and DKO-4 cells. However, the combination of Ad.mda-5 (12.5 pfu/cell) and Ad.Kras(AS) (12.5 pfu/cell) resulted in a significant decrease in growth potential in all four cell lines, further highlighting the protective role of active ras in mda-5-mediated growth suppression (Figure 6a). The relative resistance of DLD-1 to the combined growth inhibition was consistent with its decreased response to $\operatorname{Ad} . \operatorname{Kras}(\mathrm{AS})$ infection (Figure 6a).

Annexin V staining and FACS analysis confirmed that cell growth suppression induced by Ad.mda-5, Ad.Kras(AS) alone or the combination of the two viruses in human colorectal cancer cells resulted to a great extent from apoptosis (Figure 6b). Consistent with the decreased cell viability in HCT 116, Hkh2 and DKO-4 cells confirmed by MTT assays, these cells exhibited high levels of apoptotic cells (40, 47 and $44 \%$, respectively) after Ad.mda-5 plus Ad.Kras(AS) infection (Figure 6b). Although DLD-1 was relatively resistant to the combinatorial treatment, displaying only $20 \%$ apoptotic cells, this level was still statistically higher than apoptosis induced by Ad.null (7\%), Ad.mda-5 alone (12\%) or by Ad.Kras(AS) alone (10\%) (Figure 6b).

Clonal assays of DLD- 1 and DKO- 4 cells generated results consistent with those of MTT and Annexin V staining assays. At $25 \mathrm{pfu} / \mathrm{cell}$, Ad.null-infected cells gave rise to numbers of colonies comparable to that of uninfected cells (114 and $95 \%$ of that of uninfected DLD-1 and DKO-4 cells, respectively) (Figure 6c). Although Ad.mda-5 alone did not affect the colony-forming ability of mutant active K-ras expressing DLD1 cells, it induced a decline in colony numbers when applied in the presence of either Ad.Kras(AS) or PD98059 (Figure 6c). However, Ad.mda-5 alone caused a significant decrease in colony formation in DKO-4 cells lacking K-ras activity. Considering the antiapoptotic role ras plays under many conditions, the reduced colony-forming ability of Ad.Kras(AS) in both DLD-1 and DKO- 4 cells is likely due to a knockdown of $\mathrm{K}$-ras activity in DLD-1 cells, and a further inhibition of $\mathrm{H}$-ras activity in DKO-4 cells (Figure 6c).

Adenovirus-mediated expression of MDA-5 protein in these four colorectal cancer cells was determined by Western blot analysis using anti-MDA-5 antibody (Figure 7). In all four colorectal carcinoma cells, there were comparable levels of MDA-5 protein in Ad.mda-5-infected cells, and neither 

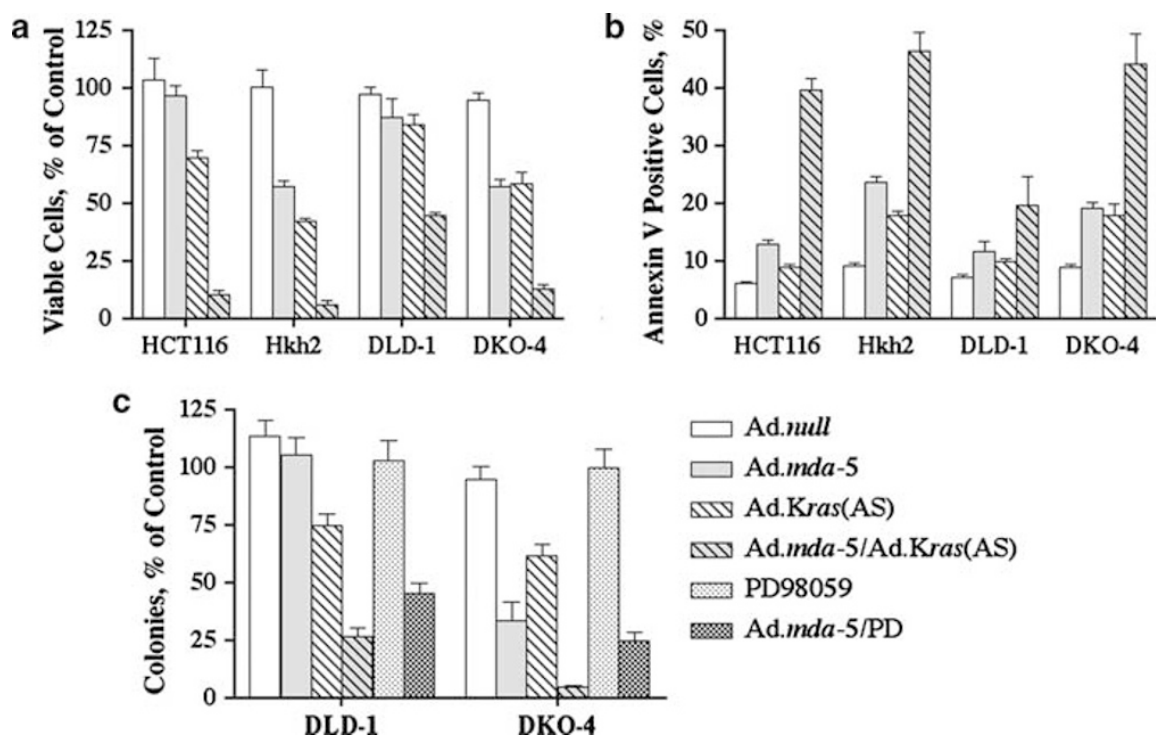

Figure 6 Blocking Ras/Raf/MEK/ERK activation using AS K-ras or PD98059 or by genetic knockout of K-Ras sensitizes mutant K-ras expressing human colorectal carcinoma cells to mda-5-induced growth inhibition and apoptosis. (a) The indicated cell type was plated at 1000 cells per well in a 96 -well plate on the previous day and were either infected with Ad.null, Ad.mda-5 or Ad.Kras(AS) at 25 pfu/cell or infected with Ad.mda-5 plus Ad.Kras(AS) at 12.5 pfu/cell of each virus. Cell viabilities were measured on the fifth day after infection. (b) The indicated cell type was plated in six-well plates ( $20000 \mathrm{cell} / \mathrm{s} / \mathrm{well}$ ) on the previous day and infected as in (a), stained with APC-labeled Annexin V and analyzed by FACS. (c) The indicated cell type was infected with Ad.null, Ad.mda-5 or Ad.Kras(AS) at 25 pfu/cell, or with Ad.mda-5 plus Ad.Kras(AS) at 12.5 pfu/cell of each virus, or with Ad. mda-5 (25 pfu/cell) in the presence of PD98059 (10 $\mu \mathrm{M})$ for $24 \mathrm{~h}$ and replated with $500 \mathrm{cells} / 60-\mathrm{mm}$ plate for colony formation assay

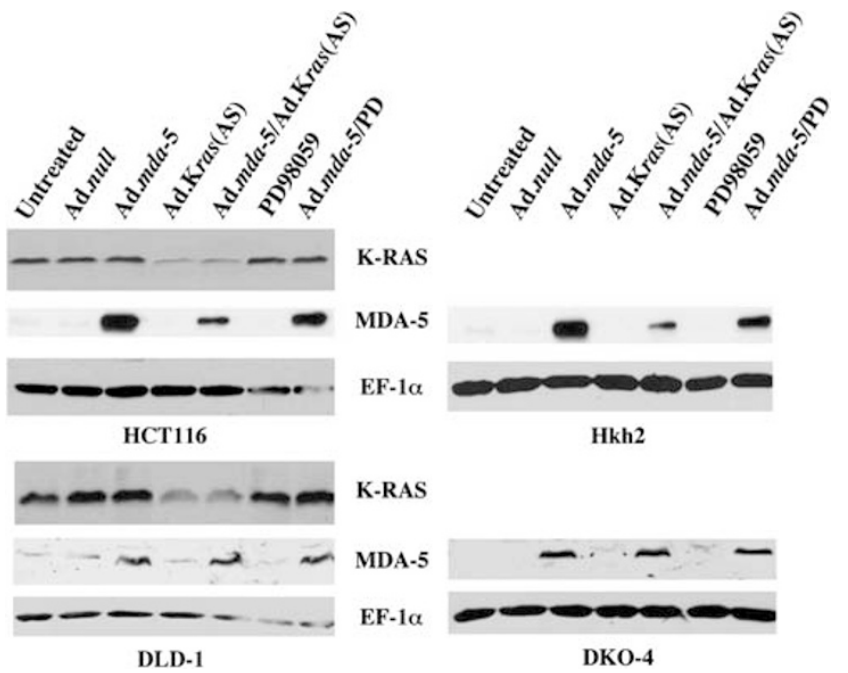

Figure 7 Expression of MDA-5 and K-Ras proteins in colorectal carcinoma cells with mutated or knocked out K-Ras following Ad.mda-5 infection in the presence or absence of Ad.Kras(AS) or PD98059. HCT 116, Hkh2, DLD-1 and DKO-4 cells were infected and/or treated as described in Figure 6, lysates were collected $48 \mathrm{~h}$ post-infection and Western blot analysis was performed using MDA-5, K-Ras and EF- $1 \alpha$ antibodies

infection with Ad.Kras(AS) nor treatment with PD98059 lead to a change in Ad.mda-5-mediated MDA-5 protein levels (Figure 7). Western blotting also confirmed the levels of K-Ras in the four colorectal cancer cells, with HCT 116 and DLD-1 expressing abundant levels of K-Ras (Figure 7), Hkh2 and DKO-4 did not contain detectable levels of $\mathrm{K}$-Ras proteins (data not shown). Ad.Kras(AS) reduced the endogenous level of K-ras by $\sim 80 \%$ in HCT 116 cells and by $\sim 40 \%$ in DLD-1 cells (Figure 7), accounting for their decreased response to mda-5-mediated killing in the presence of Ad.Kras(AS) (Figure 6a and b).

Studies have shown that different isoforms of ras, $\mathrm{K}-, \mathrm{H}$ and $\mathrm{N}$-ras can play differential and overlapping roles in cell proliferation, differentiation and apoptosis. ${ }^{26}$ We therefore employed a clone of Hkh2, HrasCL10, in which $\mathrm{H}$-ras was introduced by transfection and stably expressed, ${ }^{28,29}$ to test whether $\mathrm{H}$-ras activity could replace/restore the antiapoptotic function of K-ras in mda-5-mediated cell death. Both MTT and colony formation assays showed a statistically significant restoration of growth potential and colony-forming ability in HrasCL10 cells after Ad.mda-5 infection compared to the significantly impaired conditions in Hkh2 (Figure 8a and b). Interestingly, cell viability and cloning ability in HrasCL10 cells did not recover completely $(\sim 70 \%)$ to that in the parental mutant active K-ras expressing HCT 116 cells (Figure $8 \mathrm{a}$ and b), which could be owing to the differential protective action of $\mathrm{K}$-ras versus $\mathrm{H}$-ras or alternatively the fact that the level of $\mathrm{H}$-ras in HrasCL10 is significantly lower than that of K-ras in HCT 116 cells (Figure $8 \mathrm{c}$ ). ${ }^{28,29}$

Unlike pancreatic carcinoma cells (Figure 5b), Ad.mda-5 infection of the different colorectal carcinoma cells produced equivalent amounts of MDA- 5 protein, data shown for HCT 116, Hkh2 and HrasCL10 cells (Figure 8c). This finding argues against the possibility that the differential killing induced by Ad.mda-5 in these cells might result from differences in transduced MDA-5 protein levels (Figure $8 \mathrm{c}$ ). As shown previously, ${ }^{28,29}$ HCT 116 cells expressed high levels of K-ras, whereas there were only marginal levels of total ras protein in Hkh2 and HrasCL10 cells (Figure 8c). 

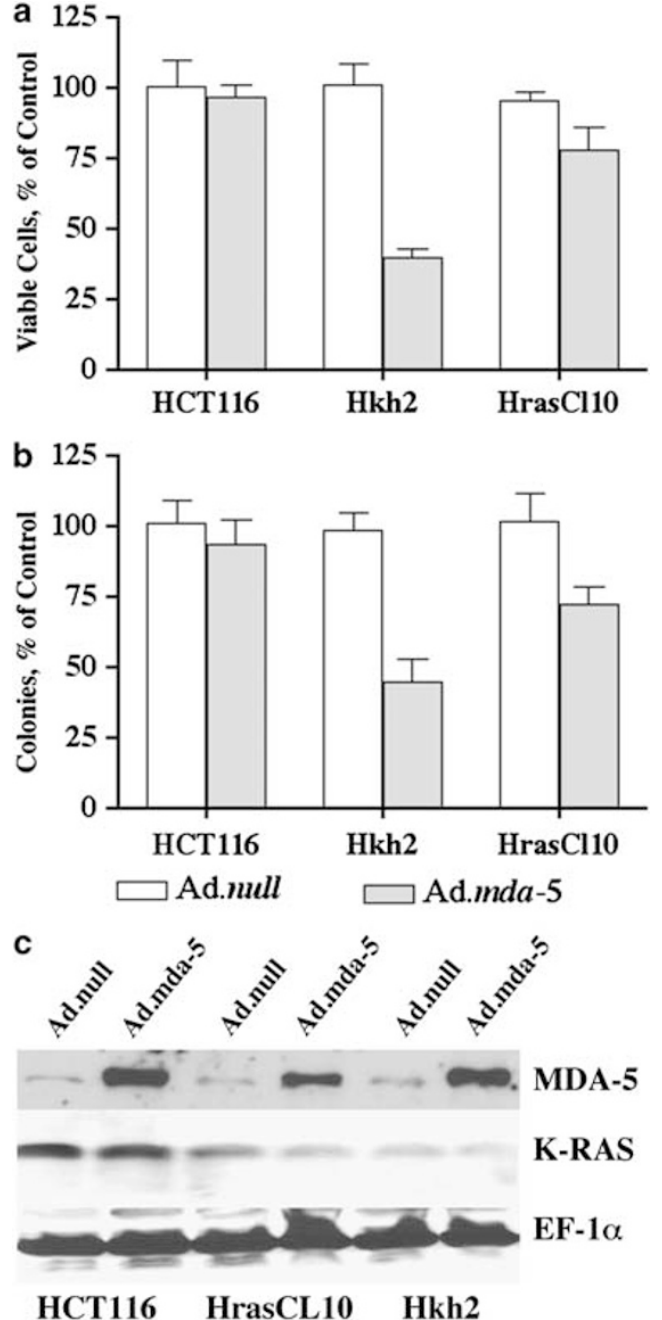

Figure 8 Stable expression of a H-ras oncogene in $\mathrm{Hkh} 2$ colorectal carcinoma cells lacking K-ras activity restores protection against mda-5-induced growth suppression in Hkh2. HCT 116, Hkh2 and HrasCL10 cells were infected with Ad.null or Ad.mda-5 at 25 pfu/cell for 2 or 5 days, and cell growth and viability were analyzed by MTT (a) or Annexin V staining assays (b), respectively. (c) Expression of MDA-5 and K-RAS proteins in HCT 116, Hkh2 and HrasCL10 cells 2 days after Ad. $m d a-5$ infection. Cells were treated as indicated, lysates collected $48 \mathrm{~h}$ post-infection and Western blot analysis was performed using MDA-5, KRas and EF- $1 \alpha$ antibodies

\section{Discussion}

We presently document a protective role of activation of the Ras/Raf/MEK/ERK pathway in mediating growth suppression and apoptosis by the novel $m d a-5$ gene in both oncogenically transformed rodent and human pancreatic and colorectal carcinoma cells. Inhibiting Ras/Raf/MEK/ERK activation by abolishing ras activity, by genetic deletion, antisense strategies or by inhibiting the ras-downstream product MEK $1 / 2$ function by its specific pharmacological inhibitor PD98059 reverses resistance to $m d a-5$-induced apoptosis. Conversely, partial restoration of ras function by stably transfecting $\mathrm{H}$-ras into colorectal carcinoma cells with genetically deleted (knockout) K-ras partially restores the antiapoptotic and prosurvival capabilities against $m d a-5$-induced proliferation inhibition and apoptosis induction.
Immortal CREF cells, which display a normal cellular phenotype, were sensitive to mda-5-induced growth suppression and induction of apoptosis. Among different oncogenetransformed CREF cells, only CREF-ras and CREF-raf demonstrated resistance to mda-5-mediated apoptosis, whereas type 5 adenovirus ( $H 5 h r), H P V-18$ and src transformation of CREF cells did not afford protection (Figure 1). In many cell types, transformation by $v$-ras or v-raf can activate MAPK pathways. ${ }^{24}$ In CREF cells, in addition to activating the MAPK pathway, transformation with the Ha-ras or V-raf oncogene also results in morphological changes and acquisition of anchorage-independent growth and tumorigenic competence in nude mice, whereas inhibition of MAPK signaling reduces anchorage-independent growth. ${ }^{15,22,23}$ The colony-forming ability of each cell line has been found to be approximately proportional to the degree of MAPK activation, ${ }^{15}$ suggesting that MAPK signaling may play a paramount role in this process. MAPK pathways can transduce a multitude of extracellular signals, leading to various cellular responses, including cell growth, differentiation, apoptosis or inflammation. ${ }^{30}$ In most cases, MAPK pathways are activated by small G proteins, such as Ras, Rac and Rap1, although they can be activated by other enzymes. In mammalian cells, there are three major MAPK pathways, MAPK/ERK, SAPK/JNK and p38 MAPK. ${ }^{30}$ Among them, the Ras/Raf/MEK/ERK pathway is positioned at the center of the signaling cascade, thereby influencing cell proliferation, differentiation and survival.

The present experiments support the hypothesis that K-ras activation is a primary contributor to the inability of $m d a-5$ to induce apoptosis in human pancreatic and colorectal carcinoma cells, tumors in which ras mutations occur at high frequencies. Not only blocking K-ras by AS or genetic deletion but inhibiting its downstream pathway MEK $1 / 2$ allows $m d a-5$ to kill K-ras mutant cancer cells. This combinatorial treatmentinduced apoptosis appears to be a synergistic effect between mda-5 and K-ras suppression. Previously, we documented that $m d a-7 / I L-24$ (melanoma-differentiation-associated gene7/interleukin-24), a tumor-specific cell death-inducing cytokine gene ${ }^{31}$ induces apoptosis specifically in $\mathrm{K}$-ras mutant human pancreatic tumor cells upon inhibition of K-ras expression. ${ }^{16,25}$ In mutant, but not wild-type, K-ras pancreatic tumor cells, mda7/IL-24 mRNA is expressed following infection with $100 \mathrm{pfu} / \mathrm{cell}$ of Ad.mda-7, whereas MDA-7/IL-24 protein and apoptosis are only induced in these cells when K-ras expression is nullified. These studies uncovered an interesting phenomenon involving a message-specific 'translational block' in pancreatic tumor cells that correlated with mutant $\mathrm{K}$-ras expression. A parallel process may also exist relative to mda-5 in mutant $\mathrm{K}$-ras pancreatic carcinoma cells, as infection with Ad.mda-5 (at doses resulting in MDA-5 protein expression in other cell types) results in mda- 5 mRNA with no detectable MDA-5 protein (Figure 5). When K-ras is inhibited using Ad.Kras(AS) in mutant, but not wild-type, K-ras pancreatic tumor cells, MDA-5 protein is detected and apoptosis is induced (Figures 4 and 5). The lack of an effect of Ad.mda-5 plus Ad.Kras(AS) in wild-type K-ras BxPC-3 cells suggests that additional pathways may be involved in modifying expression and/or retention of MDA-5 protein in different pancreatic tumor cells. Indeed, initial studies with proteasome inhibitors (data not shown) 
indicate that accelerated degradation of MDA-5 protein might also control the expression level of MDA-5 protein. The mechanism underlying suppression of specific message translation and its reversion in pancreatic tumor cells is currently under investigation.

Numerous studies have suggested that aberrant activation of the Ras/Raf/MEK/ERK pathway is a primary reason why many tumors are refractory to current therapies. Mutant active ras has been a major contributor to the inappropriate activation of this pathway, and direct inhibition of ras activity or targeting its downstream pathway, such as MEK $1 / 2$, has proven to be effective in tumor suppression. ${ }^{16}$ Ras/Raf/MEK/ ERK activation exerts multiple roles in maintaining a cell's homeostasis, including cell growth, differentiation and apoptosis. $^{30}$ The final outcome of this activation may be cell type context dependent and the contribution of different isoforms of RAS proteins, as well as the particular raf gene activated. There are three isoforms of Ras proteins identified in mammals, $\mathrm{K}-, \mathrm{H}$ - and $\mathrm{N}$-ras. Some studies have shown that $\mathrm{K}$-ras and $\mathrm{H}$-ras have distinct and overlapping specificities to downstream signaling pathways: K-ras preferentially activates Raf/MEK/ERK cascade, whereas $\mathrm{H}$-ras activates the PI3K/Akt cascade. ${ }^{7,28}$ Additionally, there is a higher mutation frequency of $\mathrm{K}$-ras than that of $\mathrm{H}$-ras in human tumors, ${ }^{17,26}$ implicating the Raf/MEK/ERK pathway as a common target for tumorigenesis. However, depending on the cell culture system used and in diverse cellular contexts, different conclusions can be drawn. For instance, some studies have documented that $\mathrm{K}$ - and $\mathrm{H}$-ras-mediated signaling pathways exert different effects on apoptosis and the K-ras downstream Raf/MEK/MAPK pathway is required for the induction of apoptosis in endometrial cells. The constitutively activated K-ras promoted apoptotic cell death, whereas the $\mathrm{H}$-ras mutant rescued rat endometrial cells from apoptosis. ${ }^{32}$ Our findings suggest that in different cell systems the ras/raf signaling pathway plays a general role in mediating resistance to Ad.mda-5-induced cell death.

We have employed both K-ras-mutated human carcinoma cells and $\mathrm{H}$-ras-activated rodent-transformed CREF cells. Both Ras isoforms provide protection against mda-5mediated killing, suggesting that both $\mathrm{K}$-ras and $\mathrm{H}$-ras can elicit an antiapoptotic function through their downstream targets, in our system most likely Raf/MEK/ERK. This is further illustrated by using an isogenic human carcinoma cell system. The parental HCT 116 cells have mutant active K-ras, and recombinant deletion of its K-RAS D13 leads to the generation of Hkh2 cells lacking K-ras activity. ${ }^{27}$ As shown earlier, Hkh2 cells also have $\sim 70 \%$ reduced basal ERK1/2, AKT and JNK1/2 activity. ${ }^{29}$ A third cell line HrasCL10 was made by genetically introducing H-RAS V12 into Hkh2 that lacks the activated $\mathrm{K}$-ras molecule. The expression of $\mathrm{H}$-ras restores the basal ERK1/2 and AKT activity. ${ }^{29}$ In our study, HCT 116 presents with high resistance to mda-5-mediated killing, which is extinguished in $\mathrm{Hkh} 2$ and partially regained in HrasCL10 cells (Figure 8). This is consistent with previous data that HrasCL10 cells show increased cell survival after radiation compared with $\mathrm{Hkh} 2{ }^{29}$ The fact that the protection regained in HrasCL10 cells against $m d a-5$-induced apoptotic effect is not $100 \%$, as is the case with HCT 116 cells, may indicate that $\mathrm{H}$-ras and $\mathrm{K}$-ras initiate different downstream pathways with different strengths. However, it is equally possible that this differential effect reflects the quantitative levels of these proteins, as the endogenous K-ras activity greatly exceeds the transfected $\mathrm{H}$-ras activity (Figure 8). These possibilities are highlighted by the fact that in HrasCL 10 cells there is no restored JNK1/2 activity as in parental HCT 116 cells. ${ }^{29}$ Further experiments are required to examine the involvement of JNK1/2 in mda-5-mediated killing.

The Ras/Raf/MEK/ERK pathway has been shown to exert its cell cycle progression effects by inducing cell cycle regulatory proteins, such as cyclin-dependent kinase inhibitors (Cdkls), including p2 $1^{\text {waf-1,Cip1, mda-6 }}$ and cyclins, and transcription factors, including NF- $\kappa \mathrm{B}, \mathrm{c}-\mathrm{Myc}$, Ets, CREB and AP-1. ${ }^{17}$ After membrane translocation initiated by Ras, activated Raf proteins associate with cell proliferation in murine and human cytokine-dependent hematopoietic cells, murine $\mathrm{NIH}-3 \mathrm{~T} 3$ fibroblasts and A10 smooth muscle cells. ${ }^{10,30}$ Src can also activate raf- 1 by phosphorylating Raf-1 at $S 338$ and $S 339 .^{10}$ However, in our study, we found that src-transformed CREF cells, CREF-src, remain responsive to mda-5-induced apoptosis. This suggests that other signaling events may also be required to activate the Raf/MEK/ERK pathway. Additionally, overexpression of Src tyrosine kinase can be proapoptotic. In human gallbladder epithelial cells, transfection of v-src sensitizes cells to taxotere-induced apoptosis in a H-ras-, Raf1-, PI3K- and PKC-independent manner. ${ }^{33}$ In NIH3T3 cells, a tyrosine phosphorylation event was involved in the negative feedback regulation of Raf- 1 . Inhibition of a Src family tyrosine kinase appears to alleviate this tyrosine kinase-mediated inhibition of Raf-1 and allows activating modification(s) of Raf1 to proceed. ${ }^{34}$ Scaffolding proteins aid the transmission of signals from Raf-1 to its downstream kinases MEK and ERK. Two proteins form complexes with members of the Raf/MEK/ ERK pathway. The kinase suppressor of Ras (KSR) forms complexes with Raf, MEK and ERK, whereas the MEK partner 1 (MP1) protein forms a complex with MEK and ERK. ${ }^{17,35}$ These complexes assure that the different members of this cascade are in the appropriate stoichiometry for efficient signal transduction. Analyzing the effect of Ad.mda-5 infection combined with inhibition of these scaffolding proteins will facilitate the dissection of the protective role of different members of the ras signaling cascade. In the present study, the inability of mda-5 to induce apoptosis in CREF-raf cells even after blocking K-ras activity by Ad.Kras(AS) approaches indicates a Ras-independent Raf activation, which was also documented in previous studies. ${ }^{34,36}$ However, the susceptibility of CREF-raf to mda-5-induced killing after MEK1/2 inhibition by PD98059 further confirms the involvement of Raf/ MEK/ERK in mda-5-mediated apoptosis. Studies are in progress to analyze the association of Ras/Raf/MEK/ERK pathway with different aspects of the apoptotic machinery involved in Ad.mda-5-induced apoptosis such as bcl-2 family members, as they have been related to Ras/Raf/MEK/ERKmodulated cell survival. ${ }^{17}$

The present studies are beginning to shed light on the role of defined signaling pathways in mediating sensitivity or resistance to $m d a-5$-induced apoptosis. A role for ras and raf in mediating resistance and an involvement of its downstream signaling component, MEK $1 / 2$, is presently illuminated. This effect has been confirmed in the context of a genetically 
homogenous CREF background as well as in human pancreatic and colorectal cancer cells. In these model systems, overexpression of ras and in the CREF context raf protects cells from mda-5-induced killing and ablating these pathways or their downstream target MEK1/2 renders these cells sensitive to apoptosis induction by mda-5. Further studies are in progress to precisely define the relevance of this important signaling pathway, Ras/Raf/MEK/ERK and the putative role of other signaling pathways in mediating response to the novel apoptosis-inducing gene mda-5. Elucidating the mechanism of action of the proapoptotic gene mda-5 will shed light on several significant processes, including interferon signaling and response, cellular differentiation, apoptosis induction and viral pathogenicity. ${ }^{3,4,37-40}$

\section{Materials and Methods}

\section{Reagents and antibodies}

All chemicals were purchased from Sigma (St Louis, MO, USA) unless indicated. PD98059 was purchased from Calbiochem (Cat\# 513000, La Jolla, CA, USA). Anti-RAF-1 (Cat\# 07-396) and EF1 $\alpha$ (Cat\# 05-235) antibody were obtained from Upstate Biotechnology (Waltham, MA, USA). c-K-RAS antibody was purchased from Oncogene Research Products (Cat\# OP24, OP24L, San Diego, CA, USA). MDA-5 antibody was produced in rabbits by repeated injections of GST-MDA-5 recombinant protein, MDA-5 ${ }_{1-335}$.

\section{Cell lines and culture conditions}

Parental CREF cells are a specific clone of Fischer F2408 cells. ${ }^{19}$ CREF cells transformed by H-ras, v-raf, v-src, HPV-18 and a specific temperature-sensitive mutant of type 5 adenovirus $H 5 h r l$ were obtained by transfecting CREF cells and isolating morphologically transformed foci. ${ }^{15,41}$ BxPC-3, PANC-1, MIA PaCa-2 and AsPC-1 pancreatic carcinoma cells were obtained from the American Type Culture Collection. Human colorectal carcinoma cells HCT 116, Hkh2 (HCT 116 without mutant K-ras), DLD-1 and DKO-4 (DLK without mutant K-ras) were provided by Dr. Bert Vogelstein, Johns Hopkins University, MD, USA. HrasCL10 (HCT 116 without mutant K-ras and expressing mutant $\mathrm{H}$-ras) were generated as described previously. ${ }^{29}$ All cells were cultured in Dulbecco's modified Eagle's medium supplemented with $5 \%$ (for parental and transformed CREF cells) or 10\% (human pancreatic and colorectal carcinoma cells) fetal bovine serum and penicillin/streptomycin (100 U/ $100 \mu \mathrm{g} / \mathrm{ml}$ ) in a $5 \% \mathrm{CO}_{2}, 95 \%$ air-humidified incubator. All cells were routinely screened for mycoplasma contamination.

\section{Adenovirus construction and infection}

The replication-incompetent adenoviruses (Ad.null, Ad.mda-5 and Ad.Kras(AS)) were constructed and amplified as described previously., Amplified virus was purified with BD Adeno- $X$ virus purification kit $(B D$ Clontech) and viral titers were determined by plaque assay in HEK 293 cells. Adenovirus was inoculated onto cells plated the previous day at the indicated m.o.i. with a minimal volume of media providing cell coverage. After $2 \mathrm{~h}$ of incubation with rotation, the infected cells were refed with fresh media. Infected cells were treated and analyzed as indicated in the figure legends.

\section{MTT cell growth and viability assays}

MTT assays were performed as described previously. ${ }^{16}$ Cells were seeded in 96-well plates and were treated next day as indicated in Results.
On the day of the assay, cells were exposed to $100 \mu \mathrm{l}$ of complete media containing $0.5 \mathrm{mg} / \mathrm{ml} \mathrm{MTT}$. After $4 \mathrm{~h}$, an equal volume of $10 \% \mathrm{SDS}-0.01 \mathrm{~N}$ $\mathrm{HCl}$ solution was added to each well and the plate was incubated at $37^{\circ} \mathrm{C}$ overnight to facilitate lysis of the MTT metabolite. Metabolic conversion of MTT by living cell was measured by A595 with Model 555 Microplate Reader (BioRad, Hercules, CA, USA).

\section{RNA isolation and Northern blot analysis}

Total RNA was extracted with Qiagen RNeasy mini kit (Qiagen, Hilden, Germany) according to the manufacturer's protocol. An EcoRI fragment $(2.5 \mathrm{~kb})$ of the mda-5 cDNA and a $0.7-\mathrm{kb}$ PCR fragment of gapdh were used as probes.

\section{Western blot analysis}

Protein extracts were prepared by lysis in $50 \mathrm{mM}$ Tris- $\mathrm{HCl}(\mathrm{pH} 7.5), 0.15 \mathrm{M}$ $\mathrm{NaCl}, 0.1 \%$ SDS, $0.1 \%$ Triton $\mathrm{X}-100,0.1 \%$ Nonidet $\mathrm{P}-40,0.5 \% \mathrm{Na}-$ deoxycholate, $1 \mathrm{mM}$ DTT and protease inhibitors (Roche) and centrifuged at 13000 r.p.m. for $15 \mathrm{~min}$ at $4^{\circ} \mathrm{C}$ Equal aliquots $(50 \mu \mathrm{g})$ of protein samples were resolved in $8 \%$ (for MDA- 5 and EF- $1 \alpha$ ) or $12 \%$ (p21 K-RAS) SDS-PAGE and transferred to a nitrocellulose membrane. Immunodetection of the Western blot was performed as described previously. ${ }^{4}$

\section{Apoptosis assays}

Annexin $\mathrm{V}$ binding assays were used to determine apoptosis induction under various treatment conditions. Briefly, $48 \mathrm{~h}$ after treatment, cells $\left(5 \times 10^{5}\right.$ cells per sample) were trypsinized, washed twice with ice-cold PBS, resuspended in $100 \mu$ l of binding buffer containing $10 \mathrm{mM}$ HEPES$\mathrm{NaOH}(\mathrm{pH} 7.4), 140 \mathrm{mM} \mathrm{NaCl}$ and $5 \mathrm{mM} \mathrm{CaCl}_{2}$, and stained with APClabeled Annexin-V (BD Biosciences, Palo-Alto, CA, USA) for $15 \mathrm{~min}$ at room temperature. Within $30 \mathrm{~min}$ after addition of $400 \mu \mathrm{l}$ of propidium iodide in the binding buffer to a final concentration of $0.5 \mu \mathrm{g} / \mathrm{ml}$, the cells were analyzed with FACS Calibur (Becton Dickinson, San Jose, CA, USA).

\section{Colony-forming (clonal) assays}

Cells were plated at $1 \times 10^{6}$ in a $10-\mathrm{cm}$ dish 1 day before infection with adenoviruses at the indicated m.o.i. At 1 day after infection, cells were trypsinized and replated at 300-800 cells/6-cm dish with $6 \mathrm{ml}$ of complete growth media. After 2 weeks, cells were fixed with $3.7 \%$ formaldehyde and stained with $5 \%$ Giemsa solution. Colonies with $\geq 50$ cells were enumerated.

\section{Acknowledgements}

The present research was supported in part by National Institutes of Health Grant GM068848, the Samuel Waxman Cancer Research Foundation and the Chernow Endowment. PBF is the Michael and Stella Chernow Urological Cancer Research Scientist in the Departments of Pathology, Urology and Neurosurgery at Columbia University Medical Center and an SWCRF Investigator.

\section{References}

1. Fisher PB, Prignoli DR, Hermo Jr. H, Weinstein IB and Pestka S (1985) Effects of combined treatment with interferon and mezerein on melanogenesis and growth in human melanoma cells. J. Interferon Res. 5: 11-22. 
2. Leszczyniecka M, Roberts T, Dent P, Grant $S$ and Fisher PB (2001) Differentiation therapy of human cancer: basic science and clinical applications. Pharmacol. Ther. 90: 105-156.

3. Kang DC, Gopalkrishnan RV, Wu Q, Jankowsky E, Pyle AM and Fisher PB (2002) mda-5: An interferon-inducible putative RNA helicase with doublestranded RNA-dependent ATPase activity and melanoma growth-suppressive properties. Proc. Natl. Acad. Sci. USA 99: 637-642.

4. Kang DC, Gopalkrishnan RV, Lin L, Randolph A, Valerie K, Pestka S and Fisher PB (2004) Expression analysis and genomic characterization of human melanoma differentiation associated gene-5, mda-5: a novel type I interferonresponsive apoptosis-inducing gene. Oncogene 23: 1789-1800.

5. Kovacsovics M, Martinon F, Micheau O, Bodmer JL, Hofmann K and Tschopp J (2002) Overexpression of Helicard, a CARD-containing helicase cleaved during apoptosis, accelerates DNA degradation. Curr. Biol. 12: 838-843.

6. Chang F, Steelman LS, Lee JT, Shelton JG, Navolanic PM, Blalock WL, Franklin RA and McCubrey JA (2003) Signal transduction mediated by the Ras/ Raf/MEK/ERK pathway from cytokine receptors to transcription factors: potential targeting for therapeutic intervention. Leukemia 17: 1263-1293.

7. Yan J, Roy S, Apolloni A, Lane A and Hancock JF (1998) Ras isoforms vary in their ability to activate Raf-1 and phosphoinositide 3-kinase. J. Biol. Chem. 273: 24052-24056.

8. Peyssonnaux C, Provot S, Felder-Schmittbuhl MP, Calothy G and Eychene A (2000) Induction of postmitotic neuroretina cell proliferation by distinct Ras downstream signaling pathways. Mol. Cell. Biol. 20: 7068-7079.

9. Vojtek AB, Hollenberg SM and Cooper JA (1993) Mammalian Ras interacts directly with the serine/threonine kinase Raf. Cell 74: 205-214.

10. Marshall CJ (1996) Cell signaling. Raf gets it together. Nature 383: 127-128.

11. Dougherty MK, Muller J, Ritt DA, Zhou M, Zhou XZ, Copeland TD, Conrads TP, Veenstra TD, Lu KP and Morrison DK (2005) Regulation of Raf-1 by direct feedback phosphorylation. Mol. Cells 17: 215-224.

12. Flotho C, Valcamonica S, Mach-Pascual S, Schmahl G, Corral L, Ritterbach J, Hasle H, Arico M, Biondi A and Niemeyer CM (1999) RAS mutations and clonality analysis in children with juvenile myelomonocytic leukemia (JMML). Leukemia 13: 32-37.

13. Hilgers W and Kern SE (1999) Molecular genetic basis of pancreatic adenocarcinoma. Genes Chromosomes Cancer 26: 1-12.

14. Minamoto T, Mai M and Ronai Z (2000) K-ras mutation: early detection in molecular diagnosis and risk assessment of colorectal, pancreas, and lung cancers - a review. Cancer Detect. Prev. 24: 1-12.

15. Su Z, Shi Y, Friedman R, Qiao L, McKinstry R, Hinman D, Dent $P$ and Fisher PB (2001) PEA3 sites within the progression elevated gene-3 (PEG-3) promoter and mitogen-activated protein kinase contribute to differential PEG-3 expression in Ha-ras and v-raf oncogene transformed rat embryo cells. Nucleic Acids Res. 29: 1661-1671.

16. Lebedeva IV, Su ZZ, Sarkar D, Gopalkrishnan RV, Waxman S, Yacoub A, Dent $P$ and Fisher PB (2005) Induction of reactive oxygen species renders mutant and wild-type K-ras pancreatic carcinoma cells susceptible to Ad.mda-7induced apoptosis. Oncogene 24: 585-596.

17. Chang F, Steelman LS, Shelton JG, Lee JT, Navolanic PM, Blalock WL, Franklin R and McCubrey JA (2003) Regulation of cell cycle progression and apoptosis by the Ras/Raf/MEK/ERK pathway (review). Int. J. Oncol. 22: 469-480.

18. Serrano M, Lin AW, McCurrach ME, Beach D and Lowe SW (1997) Oncogenic ras provokes premature cell senescence associated with accumulation of $p 53$ and p16INK4a. Cell 88: 593-602.

19. Fisher PB, Babiss LE, Weinstein IB and Ginsberg HS (1982) Analysis of type 5 adenovirus transformation with a cloned rat embryo cell line (CREF). Proc. Natl. Acad. Sci. USA 79: 3527-3531.

20. Fisher PB (1984) Enhancement of viral transformation and expression of the transformed phenotype by tumor promoters In Tumor Promotion and Cocarcinogenesis In Vitro. Mechanisms of Tumor Promotion, Slaga TJ (ed) (Boca Raton, FL: CRC Press, Inc.) pp. 57-123.

21. Babiss LE, Ginsberg HS and Fisher PB (1983) Cold-sensitive expression of transformation by a host range mutant of type 5 adenovirus. Proc. Natl. Acad. Sci. USA 80: 1352-1356.

22. Boylan JF, Shih TY, Fisher PB and Zimmer SG (1992) Induction and progression of the transformed phenotype in cloned rat embryo fibroblast cells: studies employing type 5 adenovirus and wild-type and mutant Ha-ras oncogenes. Mol. Carcinogen. 5: 118-128.

23. Su ZZ, Austin VN, Zimmer SG and Fisher PB (1993) Defining the critical gene expression changes associated with expression and suppression of the tumorigenic and metastatic phenotype in Ha-ras-transformed cloned rat embryo fibroblast cells. Oncogene 8: 1211-1219.

24. Dent P, Haser W, Haystead TA, Vincent LA, Roberts TM and Sturgill TW (1992) Activation of mitogen-activated protein kinase kinase by v-Raf in NIH 3T3 cells and in vitro. Science 257: 1404-1407.

25. Su Z, Lebedeva IV, Gopalkrishnan RV, Goldstein NI, Stein CA, Reed JC, Dent $\mathrm{P}$ and Fisher PB (2001) A combinatorial approach for selectively inducing programmed cell death in human pancreatic cancer cells. Proc. Natl. Acad. Sci. USA 98: 10332-10337.

26. Barbacid M (1987) ras genes. Annu. Rev. Biochem. 56: 779-827.

27. Torrance CJ, Agrawal V, Vogelstein B and Kinzler KW (2001) Use of isogenic human cancer cells for high-throughput screening and drug discovery. Nat. Biotechnol. 19: 940-945.

28. Caron RW, Yacoub A, Li M, Zhu X, Mitchell C, Hong Y, Hawkins W, Sasazuki T, Shirasawa S, Kozikowski AP, Dennis PA, Hagan MP, Grant $S$ and Dent $P$ (2005) Activated forms of H-RAS and K-RAS differentially regulate membrane association of PI3K, PDK-1, and AKT and the effect of therapeutic kinase inhibitors on cell survival. Mol. Cancer Ther. 4: 257-270.

29. Caron RW, Yacoub A, Mitchell C, Zhu X, Hong Y, Sasazuki T, Shirasawa S, Hagan MP, Grant S and Dent P (2005) Radiation-stimulated ERK1/2 and JNK1/2 signaling can promote cell cycle progression in human colon cancer cells. Cell Cycle 4: 456-464.

30. Dent $P$, Yacoub A, Fisher PB, Hagan MP and Grant S (2003) MAPK pathways in radiation responses. Oncogene 22: 5885-5896.

31. Fisher PB (2005) Is mda-7/LL-24 a 'magic bullet' for cancer? Cancer Res. 65: 10128-10138.

32. Ninomiya Y, Kato K, Takahashi A, Ueoka Y, Kamikihara T, Arima T, Matsuda T, Kato H, Nishida J and Wake N (2004) K-Ras and H-Ras activation promote distinct consequences on endometrial cell survival. Cancer Res. 64: 27592765.

33. Boudny V and Nakano S (2003) Src tyrosine kinase but not activated Ras augments sensitivity to taxanes through apoptosis in human adenocarcinoma cells. Anticancer Res. 23: 7-12.

34. Lee M, Kim JY and Anderson WB (2004) Src tyrosine kinase inhibitor PP2 markedly enhances Ras-independent activation of Raf-1 protein kinase by phorbol myristate acetate and H2O2. J. Biol. Chem. 279: 48692-48701.

35. Wojnowski L, Zimmer AM, Beck TW, Hahn H, Bernal R, Rapp UR and Zimmer A (1997) Endothelial apoptosis in Braf-deficient mice. Nat. Genet. 16: 293-297.

36. Zang M, Waelde CA, Xiang X, Rana A, Wen R and Luo Z (2001) Microtubule integrity regulates Pak leading to Ras-independent activation of Raf-1. Insights into mechanisms of Raf-1 activation. J. Biol. Chem. 276: 25157-25165.

37. Cocude C, Truong MJ, Billaut-Mulot O, Delsart V, Darcissac E, Capron A, Mouton Y and Bahr GM (2003) A novel cellular RNA helicase, RH116, differentially regulates cell growth, programmed cell death and human immunodeficiency virus type 1 replication. J. Gen. Virol. 84: 3215-3225.

38. Yoneyama M, Kikuchi M, Natsukawa T, Shinobu N, Imaizumi T, Miyagishi M, Taira K, Akira S and Fujita T (2004) The RNA helicase RIG-I has an essential function in double-stranded RNA-induced innate antiviral responses. Nat. Immunol. 5: 730-737.

39. Andrejeva J, Childs KS, Young DF, Carlos TS, Stock N, Goodbourn S and Randall RE (2004) The V proteins of paramyxoviruses bind the IFN-inducible RNA helicase, mda-5, and inhibit its activation of the IFN-beta promoter. Proc. Natl. Acad. Sci. USA 101: 17264-17269.

40. Sumpter Jr. R, Loo YM, Foy E, Li K, Yoneyama M, Fujita T, Lemon SM and Gale Jr. M (2005) Regulating intracellular antiviral defense and permissiveness to hepatitis C virus RNA replication through a cellular RNA helicase, RIG-I. J. Virol. 79: 2689-2699.

41. Lin J, Su Z-z, Grunberger D, Zimmer SG and Fisher PB (1994) Expression of the transformed phenotype induced by diverse acting viral oncogenes mediates sensitivity to growth suppression induced by caffeic acid phenethyl ester (CAPE). Int. J. Oncol. 5: 5-15. 